\title{
Wie gut sind Gesundheits-Apps?
}

\author{
Was bestimmt Qualität \& Risiko? Welche Orientierungshilfen gibt es? \\ Value of Health Apps?
}

\author{
How to Assess Quality and Risk? Which Decision Aids Provide Guidance?
}

Autor

Ursula Kramer

Institut

HealthOn e. V. Informations- und Bewertungsplattform für Gesundheits-Apps

Schlüsselwörter

Gesundheits-Apps, Qualitätskriterien, Risikoklassifizierung, Nachhaltigkeit, Wirksamkeit, Zielgruppenerreichbarkeit, Sicherheit

Keywords

health Apps, risk and quality assessment, decision making tool to find trustworthy health apps

Bibliografie

DOI https://doi.org/10.1055/s-0043-109130

Aktuel Ernahrungsmed 2017; 42: 193-205

(c) Georg Thieme Verlag KG Stuttgart · New York ISSN 0341-0501

Korrespondenzadresse

Dr. rer. nat. Ursula Kramer, MBA, HealthOn e. V. Informations- und Bewertungsplattform für GesundheitsApps, Emmy-Noether-Straße 2, 79110 Freiburg ursula.kramer@healthon.de

\section{ZUSAMMENFASSUNG}

Gesundheits-Apps haben in wenigen Jahren weite Teile der Bevölkerung erreicht. Ob sich mit diesen Apps die erwarteten Gesundheitsziele besser erreichen lassen und ob die Gesundheitsinformationen oder Handlungsempfehlungen der Apps unabhängig, korrekt, aktuell und damit vertrauenswürdig sind, lässt sich nicht nur für Verbraucher und Patienten, sondern auch für Ärzte, Therapeuten und Pflegekräfte, d. h. Health Care Professionals (HCP), aufgrund lückenhafter Aufklärung durch die App-Anbieter schwer einschätzen. Von staatlicher Seite ist für fast alle Apps vor Veröffentlichung in den Stores weder in Deutschland noch in den USA eine Qualitätsüberprüfung vorgesehen. Experten aus Politik und Wissenschaft fordern zum Schutz von Verbrauchern verbindliche Kriterien, die eine Qualitätseinschätzung der nicht regulierten Gesundheits-Apps erlauben. Führt man die Qualität einer App primär auf ihren Nutzen und ihre Un- bedenklichkeit für den Verbraucher zurück, rücken Aspekte wie Zielgruppenerreichbarkeit und Zielgruppenorientierung einer App als qualitätsbestimmende Faktoren in den Vordergrund. Nur wenige Apps erreichen relevante Nutzergruppen und werden nachhaltig genutzt. Unter dem Aspekt der Patientensicherheit sind große Unterschiede im Nutzungsrisiko einer Gesundheits-App erkennbar. In diesem Kontext wird die 4-stufige Risikoklassifizierung vorgestellt, die auf der Grundlage der Analyse von über $6000 \mathrm{Ge}$ sundheits-Apps empirisch entwickelt wurde. Außerdem wird am Beispiel der Qualitäts- und Transparenzkriterien des HealthOn-Ehrenkodex gezeigt, wie sich GesundheitsApps einschätzen lassen im Hinblick auf folgende Aspekte: Individuelles Nutzungsrisiko für ihren Anwender, Sachverständigkeit des Unterstützungsansatzes, Unabhängigkeit der gesundheitsbezogenen Inhalte, Schutz der mit der App erfassten persönlichen Nutzungs- und Gesundheitsdaten, Möglichkeit des Nutzers im Haftungsfall Verbraucherrechte durchsetzen zu können.

\section{ABSTRACT}

Within only few years Health Apps have reached a wide range of healthy and chronically ill people worldwide. Based on the huge number of health and medical apps available in the big app stores and the lack of general regulation and quality control in Germany and the US there is a high need for orientation. As information disclosed by most app developers is in general incomplete, transparency is bad. That is why Health Care Professionals (HCP) as well as patients are looking for criteria to assess quality and trustworthiness of health apps in order to understand the drivers for risk and avoid impairment due to safety and data rights infringements or wrong or biased health information. Quality of health apps derives primarily from value and safety for its users and correlates with aspects of reach and user orientation as well. Only a minority of health and medical apps offered in German language are so far able to reach relevant download numbers, retention rates are in general low which questions the value of apps in reaching long-term health goals. Risks associated with health apps vary remarkably dependent on the supporting functions an app delivers and the health goals users are following with a 
certain app. A 4-step risk scale developed empirically based on the analyses of more than 6000 Health Apps and a set of quality and transparency determining criteria (HealthOn-Ehrenkodex) are introduced as tools to support HCP as well as patients to make informed decisions which health app to recommend or to choose best.

\section{Einführung}

Mit der Vielfalt der Apps und Wearables, die über Sensoren unterschiedliche Vital- und Körperfunktionen aufzeichnen von Schlaf-wach-Rhythmus über Stress, bis zu Herzrhythmus, Körperhaltung, Blutdruck, Muskeltonus etc. verbindet sich die Hoffnung vieler Menschen, ihr gesundheitliches Wohlergehen messen und durch eigene Zutun steuern zu können [1, 2]. Dies ist vermutlich ein Grund für den Siegeszug der GesundheitsApps und Wearables, die in wenigen Jahren relevante Nutzerzielgruppen erreicht haben [3]. Tatsächlich entscheidet das individuelle Verhalten des Einzelnen maßgeblich über die Anzahl der zu erwartenden, gesunden Lebensjahre, und zwar in viel stärkerem Maße als Faktoren wie genetische Veranlagung, soziales Umfeld, die medizinische Versorgung oder Umweltfaktoren $[4-6]$ ( $\triangleright$ Abb. 1$)$.

Gerade weil die Hauptkrankheitslast in den Industrienationen durch Krankheiten verursacht wird, die eine hohe verhaltensbedingte Risikokomponente aufweisen [7], z. B. Rückenschmerz, Herzkreislauferkrankungen, Depression, Lungenkrebs, Diabetes, liegen große Hoffnungen auf neuen Versorgungsansätzen, die über digitale Gesundheitsanwendungen die Früherkennung von Risikofaktoren verbessern und über individualisierte, motivierende Unterstützungsansätze die Selbstaktivierung des Einzelnen stärken [8]. Auf diesem Weg könnten digitale Gesundheitsanwendungen wie Apps zukünftig dazu beitragen, das Gesundheitssystem präventions- und nutzerzentriert umzubauen.

Darüber hinaus eröffnet Digitalisierung in der Gesundheitsversorgung auch neue Möglichkeiten der Partizipation, d.h. der Beteiligung des Einzelnen an Entscheidungsprozessen. Verbraucher können Angebote, Leistungen und Leistungserbringer mit Hilfe digitaler Gesundheitsanwendungen bewerten, Informationen empfehlen und Erfahrungen mit anderen Betroffenen z.B. in sozialen Medien teilen. Das könnte einerseits den Zugang zu gesundheitsförderlichen Angeboten erleichtern und gleichzeitig durch Wettbewerb die Qualität und Patientenorientierung der Angebote fördern [8].

Innovative Versorgungsansätze im Bereich der digitalen Gesundheit, z. B. Gesundheits- und Medizin-Apps und deren Risiken und Chancen haben für gesundheitspolitisch Verantwortliche daher eine ebenso hohe Brisanz erlangt, wie die Frage nach den erforderlichen Maßnahmen und Rahmenbedingungen, um diese in zukünftige Versorgungslandschaften zu integrieren. Dies zeigt sich an der wachsenden Zahl wissenschaftlicher Studien zum Einsatz von Digital Health [9] sowie an der wachsenden Zahl von Konsultationen [10], Gutachten und Stellungnahmen zur Standortbestimmung im Bereich Digital Health auf europäischer sowie nationaler Ebene [11-13]. Darin fordern Ex-

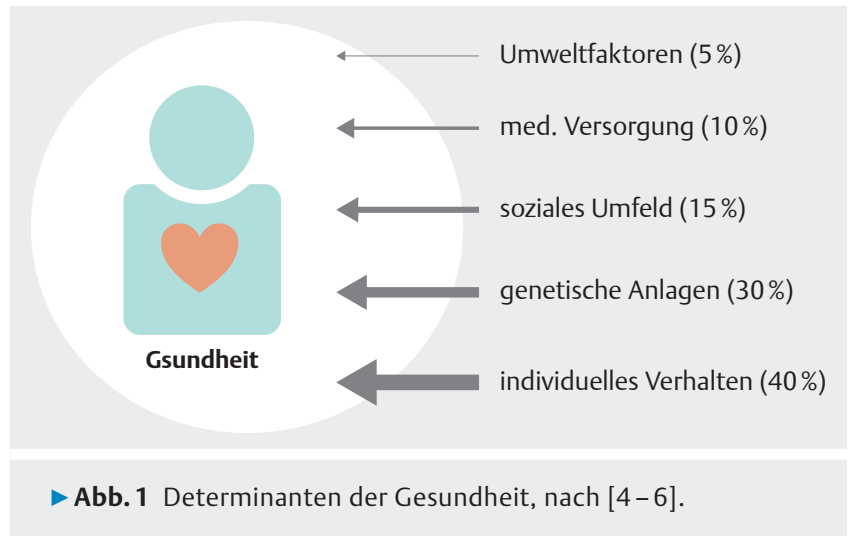

perten als Reaktion auf die hohe Verbrauchernachfrage und das weitgehende Regulierungsvakuum bessere Orientierungshilfen sowohl für Verbraucher und Patienten, Leistungserbringer und Kostenträger als auch für gesundheitspolitische Akteure.

\section{Abgrenzung Gesundheits-Apps, Medizin-Apps, Apps als Medizin-Produkt}

Häufig werden die Begrifflichkeiten Gesundheits-Apps, Medizin-Apps, App als Medizinprodukt unscharf genutzt oder sogar synonym verwendet. Auch wenn es keine verbindliche Definition gibt [14], hat sich die folgende Systematik bewährt: Danach lassen sich die mittlerweile über 220000 sog. „Gesundheits-Apps“, die in den beiden großen App-Stores derzeit verfügbar sind [15] sinnvoll einteilen nach den Gesundheitszielen, die die App verfolgt und den Nutzerzielgruppen, für die sie konzipiert ist ( $\triangleright$ Tab. 1 ).

Gesundheits-Apps richten sich demnach an gesunde Nutzer, die sich mit der App bei einem gesundheitsförderlichen Lebensstil unterstützen wollen, die ihre Ressourcen - zur Entspannung, zum besseren Verständnis von Gesundheit bzw. Krankheit, zu einer gesunden, bewegten Lebensführung - mithilfe einer App stärken wollen. Im englischen Sprachraum werden diese Apps auch als Health-Apps bezeichnet.

Medizin-Apps sind im Gegensatz dazu für Patienten oder deren Angehörige bestimmt, die nach einer Unterstützung suchen, um ihren Alltag, z. B. mit einer chronischen Krankheit, besser bewältigen zu können. Diese Apps sind darauf ausgerichtet, die „Selbstbefähigung“ von Betroffenen zum Krankheitsmanagement zu stärken. Auch Apps, die Angehörige von Heilberufsgruppen (Ärzte, Pflegekräfte, Therapeuten) z. B. mit Nachschlagewerken, Dosierungsrechnern, medizinischen Entscheidungshilfen in ihrem Praxis- oder Klinikalltag unterstützen, zählen zu den Medizin-Apps. Im englischen Sprachraum werden Medizin-Apps auch als Medical-Apps bezeichnet.

Apps als Medizinprodukte (MPG §3): Sowohl GesundheitsApps als auch Medizin-Apps können als Medizinprodukte in Verkehr gebracht werden, wenn der Anbieter sie mit einer sog. „primären medizinischen Zweckbestimmung“ anbietet. Dies ist erforderlich, wenn die App die Vermeidung, Diagnose oder Therapie von Krankheiten unterstützen will. Diese Apps müssen - je nach Risikoklasse der App - in einem mehr oder weniger aufwendigen Prozess ein EU-Konformitätsverfahren durchlaufen (Anhang IX der Richtlinie 93/42/EWG). Dann dürfen diese 
- Tab. 1 Gesundheits-App, Medizin-App, Medizinprodukt: Klassifizierung und Relevanz [15].

\begin{tabular}{|l|l|l|l|l|}
\hline & Gesundheits-Apps & Medizin-Apps & Apps als Medizinprodukt \\
\hline $\begin{array}{l}\text { Inhalte bzw. Unterstützungs- } \\
\text { funktionen }\end{array}$ & $\begin{array}{l}\text { gesundheitsbezogene } \\
\text { Inhalte }\end{array}$ & $\begin{array}{l}\text { medizinische } \\
\text { Inhalte }\end{array}$ & $\begin{array}{l}\text { medizinische } \\
\text { Inhalte }\end{array}$ & $\begin{array}{l}\text { Erfassung oder Auswertung von } \\
\text { Gesundheits-/Messdaten }\end{array}$ \\
\hline Zielsetzung & $\begin{array}{l}\text { Gesundheitsaufklärung, } \\
\text { Ressourcenstärkung }\end{array}$ & $\begin{array}{l}\text { Krankheitsbewäl- } \\
\text { tigung/Selbshilfe }\end{array}$ & $\begin{array}{l}\text { Entscheidungs- } \\
\text { hilfe }\end{array}$ & Diagnose \& Therapie \\
\hline Nutzerzielgruppe & Gesunde & $\begin{array}{l}\text { Patienten \& An- } \\
\text { gehörige }\end{array}$ & $\begin{array}{l}\text { Ärzte \& Pflege- } \\
\text { kräfte }\end{array}$ & Patienten, Ärzte \& Pflegekräfte \\
\hline Regulierung/Zulassung & nein & nein & nein & §3 Medizinproduktegesetz/ \\
\hline $\begin{array}{l}\text { Anzahl verfügbarer Apps, } \\
\text { deutschsprachig Google Play, } \\
\text { März 2016 }\end{array}$ & rd. 6400 & rd. 2100 & 10 \\
\hline
\end{tabular}

Apps ein CE-Kennzeichen tragen [16]. Gesundheits- und Medizin-Apps, die als Software ein Messgerät oder einen Sensor in einem tragbaren Gerät oder einem Armband, einem sog. Wearable, steuern oder dessen Anwendung beeinflussen, werden automatisch derselben Risikoklasse zugerechnet wie das Produkt, das sie steuern. Medizin-Apps auf Smartphones und Tablets können meistens der Risikoklasse I zugeordnet werden. Bei Medizinprodukten zur Diagnose oder Kontrolle von Vitalfunktionen, z. B. der Herzfunktion, kann eine Einstufung in höhere Risikoklassen Ila oder Ilb erforderlich sein.

Hinweis: Die inhaltliche Qualität, der Nutzen für den Anwender, die Bedienbarkeit der App oder auch der Umgang mit den Nutzerdaten und der Schutz der Privatsphäre des Nutzers sind nicht Gegenstand dieser Prüfung. CE-gekennzeichnete Apps sind demnach nicht von vorneherein besser oder vertrauenswürdiger als andere Apps. Das sollten Verbraucher wissen.

\section{Gesundheits-Apps: qualitätsbestimmen- de Parameter}

Um Nutzen schaffen zu können für den Anwender, muss eine Gesundheits-App eine Reihe von Grundvoraussetzungen erfüllen, dazu zählen:

- Zielgruppenerreichbarkeit. Haben Verbraucher bzw. Patienten einen Zugang zum gesundheitsförderlichen Angebot und können sie die App nutzen, insbesondere jene, die einen Bedarf haben?

- Nachhaltigkeit. Lassen sich Gesundheitsziele mithilfe der App nachhaltig erreichen? Kann die App das Interesse der Nutzer über längere Zeiträume binden?

- Wirksamkeit: Lässt sich die von der App ausgelobte, gesundheitliche Zielsetzung erreichen und lässt sich dies mit wissenschaftlich anerkannten Methoden überprüfen?

- Sicherheit. Ist die Anwendung der App für den Nutzer sicher bzw. wird eine gesundheitsförderliche Maßnahme, z. B. Medikamentenmanagement, durch Nutzung der App sogar sicherer?
Der Begriff Gesundheits-Apps schließt im Folgenden auch Medizin-Apps sowie CE-gekennzeichnete Gesundheits- und Medizin-Apps ein.

\section{Qualität aus Nutzersicht}

Nutzer von Gesundheits- und Medizin-Apps orientieren sich bei ihrer Suche nach einer hilfreichen App zunächst an den Angaben des App-Anbieters sowie am Urteil anderer App-Nutzer [1]. Empfehlungen von Therapeuten spielen bislang selbst bei App-Nutzern mit chronischen Grunderkrankungen kaum eine Rolle [2].

Bis zu 8 Bildschirmansichten der App und ein Beschreibungstext, mit dem der App-Anbieter darüber informiert, was die App können soll, stehen App-Nutzern als erste Informationsquelle zur Verfügung. Wenn die App, die kostenlos ist [1], die erwartete Hilfe verspricht, die Bild- und Farbwelten ansprechen und die Menüführung übersichtlich erscheint, wird die App genauer betrachtet. Bevor der Nutzer die App herunterlädt und selbst prüft, ob die App einfach und intuitiv anzuwenden ist, orientiert er sich am Urteil anderer Nutzer [1], die bereits Erfahrungen gesammelt haben: Wie gut bewerten diese Nutzer eine App auf einer Skala von 1 bis 5 Sternen, über welche Probleme berichten sie in Kommentaren, wie groß ist das Interesse an dieser App, d. h. wie oft wurde sie schon heruntergeladen? Die Anzahl der Downloads wird in Google Play ausgegeben, der App-Store von Apple informiert darüber nicht.

Weil diese Bewertungen in hohem Maße die Entscheidung anderer Nutzer beeinflussen, sind sie das Ziel von Manipulationsversuchen, d.h. App-Anbieter versuchen positive Bewertungen zu kaufen. Deshalb ist es wichtig, die Zahl der Nutzerbewertungen in Relation zu den Downloads zu sehen, die eine App erreicht. Hat eine App viele positive Bewertungen aber nur wenige Downloads, kann das Nutzerurteil in Zweifel gezogen werden. Tausende Nutzerbewertungen lassen sich hingegen nur mit unverhältnismäßig hohem finanziellen Aufwand manipulieren.

Verwalten Nutzer mit einer App persönliche Gesundheitsdaten, ist es ihnen wichtig, dass diese nicht in falsche Hände geraten oder zu ihrem Nachteil von Dritten unbefugt verwendet werden [1]. Im Gegensatz dazu steht die Beobachtung, 


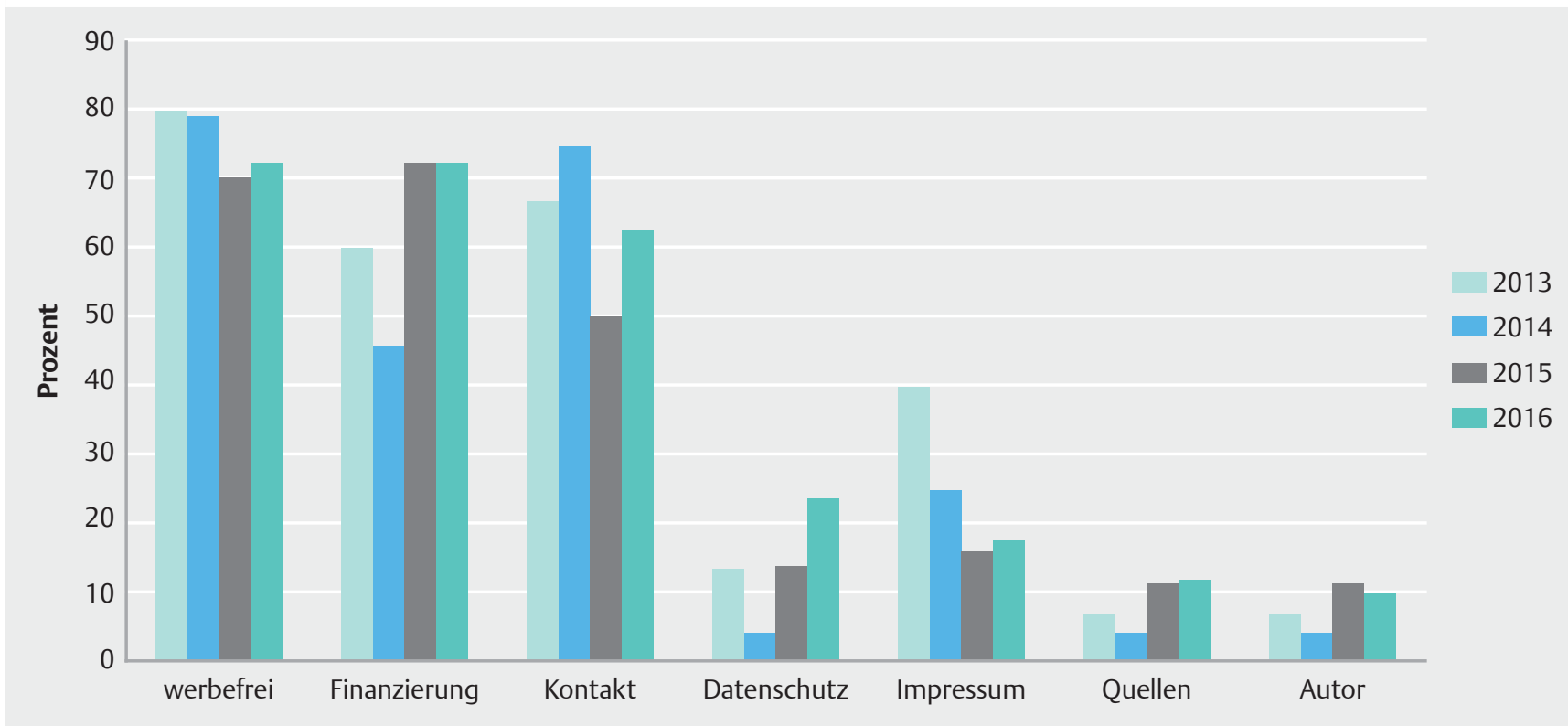

- Abb. 2 Diabetes-Apps: Vergleich 2013/2014/2015/2016. Erfüllung des HealthOn-App-Ehrenkodex in [\%]. Deutschsprachige, kostenlose Diabetes-Apps in Google Play $(n=15 / 24 / 44 / 51)$ [17].

dass z.B. nur etwa jede vierte Diabetes-App überhaupt mit einer Datenschutzerklärung informiert, ob und wie sie die Nutzerdaten schützt [17]. Das hält Anwender offensichtlich nicht davon ab, Apps zu nutzen. Erkennen Anwender persönliche Vorteile in der Weitergabe ihrer Nutzungsdaten, z. B. in Form von Bonuszahlungen der Krankenkasse [18], ist jeder Dritte zur Weitergabe dieser Daten bereit.

Ein Fragebogen, der die aus Nutzersicht relevanten Kriterien erfasst und mit den Nutzerbewertungen in den Stores korrelieren soll, wird derzeit in Pilotprojekten validiert [19, 20].

\section{Qualität aus Sicht der Kostenträger und Leistungs- erbringer}

Der Qualitätsbegriff, den Verantwortliche auf kassenfinanzierte, therapeutische oder präventive Maßnahmen anwenden, sieht die Einhaltung definierter Kriterien vor [21, 22]. Auch an gesundheitsförderliche Maßnahmen, die von öffentlichen Institutionen entwickelt werden, z.B. der Bundeszentrale für gesundheitliche Aufklärung [BZgA], werden strenge Qualitätskriterien angelegt [23-25]. Dazu zählen u.a.

1. wissenschaftlicher Nachweis der Wirksamkeit, die sog. Evidenz

2. überprüfbare Qualität der gesundheitsbezogenen Inhalte [DISCERN, Gute Praxis Gesundheitsinformationen]

DISCERN ist ein wissenschaftlich international anerkannter Katalog zur Überprüfung der methodischen Qualität von Patienteninformationen. Er wurde vom Institut für Epidemiologie, Sozialmedizin und Gesundheitssystemforschung der Medizinischen Hochschule Hannover und dem Ärztlichem Zentrum für Qualität (ÄZQ) aus dem Englischen ins Deutsche übertragen und ist verfügbar über http://www.discern.de.
Gute Praxis Gesundheitsinformation ist ein gemeinsames Grundsatzpapier verschiedener Institutionen des Gesundheitswesens zur Festlegung von Qualitätskriterien für Patienteninformationen, das folgende Anforderungen stellt [26]:

- Literaturbasierte Inhalte: Gibt es keinen Nachweis aus Studien, mit dem sich Aussagen belegen lassen, dann ist explizit auf die fehlende Evidenz hinzuweisen.

- Zielgruppenorientierte Inhalte: Alle Informationen sind verständlich, auch für Bürger ohne hohen Bildungsabschluss oder mit anderem Sprach- und Kulturhintergrund.

- Entwicklung der Inhalte unter Beteiligung von Patienten bzw. Bürgern. Vertreter aus dem Kreis, an den sich Informationen richten, sollen in die Entwicklung der Gesundheitsinformationen eingebunden sein.

- Realistische Darstellung des Wissens sowie der Grenzen dieses Wissens und Unterstützung des Nutzers durch Hinweise auf weiterführende Beratungs- und Kontaktangebote.

- Relevanz der dargestellten Ergebnisse. Ergebnisse, die für den Nutzer relevant sind, stehen im Fokus, d. h. z. B. wie wirkt sich - die Nutzung einer App - auf die Beschwerdebzw. Krankheitswahrscheinlichkeit und auf die Lebensqualität aus? Der Nutzer wird informiert über alle Begleitumstände der Nutzung des Angebots, d. h. welche körperlichen, seelischen oder finanziellen Belastungen sind mit der Nutzung der Anwendung verbunden.

- Kommunikation von Nutzen und Schaden einer Applikation. Sie erfolgt so, dass Patienten Nutzen und Schaden möglichst realistisch einschätzen können.

- Offenlegung von Interessenkonflikten: Alle Umstände sind offenzulegen, d. h. Verfasser sowie Finanzierungsquellen sind zu nennen, um mögliche Interessenkonflikte aufklären zu können. 
Diese Anforderungen an die Qualität lassen sich grundsätzlich auch auf Gesundheits- und Medizin-Apps anwenden. Untersuchungen des deutschsprachigen Angebots in verschiedenen, aus Public-Health-Sicht relevanten Indikationen zeigen selbst in den formalen, einfach überprüfbaren Angaben [14, 27] große Lücken, d.h. App-Anbieter klären in der Regel unzureichend auf [17], sodass die Einschätzung von Qualität und Vertrauenswürdigkeit für die meisten Gesundheits-Apps nicht ohne Weiteres möglich ist ( $\mathbf{A} \mathbf{b b} \mathbf{b} \mathbf{2}$ ).

\section{Wirksamkeit}

Die Frage nach der Wirksamkeit von Gesundheits- und MedizinApps und dem daraus ableitbaren Nutzen für den Einzelnen und die Gesellschaft wird derzeit sehr intensiv erforscht [11-13]. Dabei wird zum einen beleuchtet, ob und wenn ja wie Apps die psychologischen Modelle zur Veränderung des Gesundheitsverhaltens aufgreifen [28], ob und wie sie das für die Anwendung relevante, evidenzbasierte, medizinische Wissen berücksichtigen, und dem Nutzer überprüfbar helfen können, die ausgelobte, gesundheitliche Zielsetzung zu erreichen. Die meisten Apps weisen sowohl in der Methodik ihrer Unterstützungskonzepte als auch in der Evidenzgrundlage ihrer Inhalte große Schwächen auf und bleiben den Nachweis der nachhaltigen Wirksamkeit bisher schuldig [11-13].

Bisher bieten $50 \%$ der weltweit verfügbaren, relevanten Gesundheits-Apps als Unterstützungsansatz lediglich die Vermittlung von Informationen [29]. Jede zehnte App ist in der Lage, Daten auszuwerten, die über Messgeräte oder Sensoren in sog. Wearables erfasst werden. 65 \% der Apps bieten eine direkte Verbindung zu sozialen Medien, über die Daten mit Dritten einfach geteilt werden können. Bisher unterstützen lediglich $2 \%$ der Apps den Austausch von Daten mit medizinischen Leistungserbringern über sog. digitale Patientenakten [29]. Dies zeigt, dass Apps bisher auch in den USA kaum in der gesundheitlichen Regelversorgung angekommen sind, Apps werden bisher weitgehend von Verbrauchern und Patienten im Selbstmanagement ohne Einbindung von Therapeuten genutzt. Gründe dafür sind u. a.

- fehlende oder unklare verbindliche Rahmenbedingungen zum Datenaustausch, z. B. Hürden im Datenaustauschs durch unzureichende Interoperabilität der technischen Systeme, fehlende Empfehlungen zur Umsetzung der Datenschutzstandards im Praxisalltag sowie zur Nutzung der von Patienten mit Apps und Wearables erfassten Daten [11-13]

- offene Fragen der Honorierung und Haftung im Falle der Nutzung der von Patienten generierten Daten aus Apps und Wearables im therapeutischen Entscheidungsprozess [11-13]

- fehlende Methoden zum Nutzen- und Qualitätsnachweis von Gesundheits- und Medizin-Apps [11 - 13]

Gesundheitswissenschaftler und Versorgungsforscher verschiedener Disziplinen arbeiten derzeit in Arbeitsgemeinschaften und Kommissionen von Fachgesellschaften an der Entwicklung von Methoden, um Wirksamkeit und Nutzen von Gesundheits- und Medizin-Apps als Interventionen im therapeutischen und präventiven Gesundheitskontext zu evaluieren [30]. Die hohe Dynamik, mit der sich mobile Datennetze und Betriebssysteme sowie Sensoren in Smartphones und Wearables entwickeln, stellt hohe Anforderungen an Apps, die störungsfrei funktionieren sollen in technischen Umgebungen, die sich schnell verändern. Im Gegensatz dazu stehen die für die Durchführung wissenschaftlicher Langzeitstudien zum Wirksamkeitsund Nutzennachweis geforderten, definierten Rahmenbedingungen [31]. Apps sind außerdem in der Regel nur ein Baustein im Rahmen häufig komplexer Therapie- oder Präventionsinterventionen. Nutzen- und Wirksamkeitseinschätzungen von Gesundheits- und Medizin-Apps erfordern daher die Modifikation etablierter Evaluationsmethoden [32], um den spezifischen Charakter digitaler Interventionen zu berücksichtigen.

Die Nutzung und Kassenerstattung von Apps im Rahmen von Pilotprojekten, in denen die Wirksamkeit von Apps wissenschaftlich evaluiert wird, sind ein Versuch, sich methodisch zu nähern. Beispiele sind die App-Tinnitracks sowie die beiden Online-Programme Caterna Sehschulung sowie eine Online-Stottertherapie [33]. Nach den Ergebnissen einer aktuellen Studie mit der App-Tinnitracks führt deren Nutzung bei Betroffenen offensichtlich nicht zur erhofften Verbesserung der Tinnitus Belastungssituation [34].

Kassenfinanzierte Apps können auf Basis geltenden Rechts (§12 SGB V) nur dann dauerhaft als Baustein in Präventionsund Therapiekonzepten genutzt und erstattet werden, wenn sie zweckmäßig sind und deren Wirkung wissenschaftlich nachgewiesen ist.

\section{Zielgruppenerreichbarkeit}

Die Voraussetzungen von Gesundheits-Apps, große Bevölkerungsgruppen über Schicht- und Altersgrenzen hinweg in ihren Lebens- und Arbeitswelten zu erreichen, sind durch die weite Durchdringung moderner Industriegesellschaften mit Smartphone äußerst günstig [3]. Grundsätzlich ist das Interesse an Gesundheits-Apps und Wearables groß [35], jeder Dritte nutzt sie, um Gesundheitsdaten aufzuzeichnen, wenngleich Alter, Einkommen und Bildungsgrad die Erreichbarkeit beeinflussen [36, 37]. Die Möglichkeiten, Gesundheitsinformationen selbstbestimmt zu nutzen, werden grundsätzlich stark beeinflusst durch die Gesundheitskompetenz (Health Literacy) der Nutzerzielgruppen, das gilt für gesundheitsförderliche Interventionen im Allgemeinen $(38,39]$ sowie für GesundheitsApps im Besonderen [40]. Gerade Zielgruppen mit geringer Health Literacy haben einen hohen Bedarf für gesundheitsförderliche Maßnahmen [41], sie mit Apps zu erreichen, die auf die speziellen Anforderungen dieser Nutzer ausgerichtet sind, ist besonders herausfordernd [42, 43]. Grundsätzlich entscheidet sich die Qualität einer Gesundheits-App in hohem Maße daran, ob sie die Fähigkeiten, Einschränkungen und Erwartungen der Nutzerzielgruppe kennt und berücksichtigt, um von dieser verstanden (z. B. Sprache, Menüführung etc.) und einfach bedient werden zu können (z.B. Schriftgröße, Funktionen etc.) [44]. Untersuchungen zeigen in diesem Punkt große Schwächen des vorhandenen Angebots, das insbesondere von Nutzern mit geringer Gesundheits- und Technikkompetenz nur unzureichend genutzt werden kann $[45,46]$. 
Gelingt es einer App mit ansprechendem Design und hoher Anwenderfreundlichkeit das Interesse vieler Nutzer zu wecken, schlägt sich dies in der Regel auch in einer hohen Anzahl an Downloads nieder. Wie aktuelle Untersuchungen des App-Angebots zeigen, erreicht derzeit nur etwa jede 10. deutschsprachige Gesundheits- und Medizin-App mehr als 50000 Downloads [47], d.h. die meisten Apps liegen weitgehend unbeachtet und ungenutzt in den App-Stores. In der Vielzahl dieser unbedeutenden Apps die wenigen, relevanten Gesundheits-Apps zu finden, ist schwierig und fordert von Suchenden ein hohes Maß an Geduld.

Trotzdem knüpfen auch viele Patienten hohe Erwartungen an Gesundheits-Apps. Wie eine aktuelle Befragung von Diabetikern zeigt, ist nur jeder Fünfte skeptisch und glaubt nicht daran, dass das Diabetesselbstmanagement mit App erfolgreicher verläuft [2]. Auch viele Verbraucher, die sich für Ernährung und Gewichtskontrolle interessieren, suchen in den App-Stores nach Unterstützung: Unter den deutschsprachigen Top-Apps mit besonders vielen Downloads und Nutzerbewertungen finden sich daher viele Apps, die vorgeben, bei der Kontrolle des Körpergewichts zu helfen oder Nutzer bei der gesunden Ernährung zu unterstützen [48].

\section{Nachhaltigkeit}

Für die Erreichung von Gesundheitszielen sind in der Regel langfristige Änderungen im Lebensstil bzw. dem Selbstmanagement von Krankheiten erforderlich [49]. Daher misst sich die Qualität einer Gesundheits-App auch an ihrer Fähigkeit, Nutzerzielgruppen langfristig binden zu können.

Marktforschungsanalysen zeigen, dass das Interesse von Nutzern an Apps in der Regel nach wenigen Wochen nachlässt [50]. Das gilt auch für Apps, die in den Kategorien Gesundheit \& Fitness sowie Medizin angeboten werden:

- etwa die Hälfte dieser Apps erreicht eine durchschnittliche Nutzungsdauer von 30 Tagen

- etwa ein Drittel schafft es 90 Tage, das Interesse ihrer Nutzer zu binden

Mittlerweile erfassen Analysetools die Nutzungsdaten von über 800000 Apps weltweit. Die 30-Tage-Nutzung bzw. Retentionsraten für Apps im Allgemeinen sind insgesamt rückläufig. Denn obwohl der Unterstützungsumfang vieler Apps immer umfassender wird, wächst mit der Anzahl der verfügbaren Apps weltweit auch der Wettbewerb und damit der Kampf um die Gunst der Nutzer. Dies erklärt den allgemeinen Rückgang der sog. Retentionsraten. Anders in der Kategorie Gesundheit \& Fitness: Hier sind die 30-Tage-Retentionsraten mit knapp $50 \%$ konstant geblieben [51].

Ärztliche Empfehlung können die Retentionsraten von Gesundheits-Apps um ca. $10 \%$, bei reinen Fitness-Apps sogar um $30 \%$ erhöhen [29]. 59\% der Apps, die Ärzte ihren Patienten in den USA über App-Plattformen wie AppScript empfehlen bzw. verordnen können, erreichen eine durchschnittliche Nutzungsdauer von 30 Tagen, bei den Fitness-Apps sind es sogar 79\%.

Im Gegensatz dazu stehen die Ergebnisse einer aktuellen Befragung von Diabetikern zur Nutzungshäufigkeit und -dauer von Diabetes-Apps. Über $70 \%$ geben an, die Apps täglich zu nutzen, über $60 \%$ bereits länger als 6 Monate [2].

\section{Sicherheit}

Auch für Apps als digitale Interventionen sollten die Grundsätze der Patientensicherheit bzw. des Verbraucherschutzes gelten: Durch die Nutzung einer guten Gesundheits-App darf der Anwender keinen Schaden erleiden. Der Nutzer darf z. B. nicht im falschen Glauben an eine unwirksame App andere wirksame therapeutische Interventionen vernachlässigen oder durch Fehl- oder Falschinformationen zu Schlussfolgerungen gelangen, die sich negativ auf seine Gesundheit auswirken, z.B. durch Verunsicherung im Falle von falsch positiver Diagnose, durch Verharmlosung im Falle von falsch negativer Diagnose oder durch ein Fehlverhalten, das auf eine falsche medizinische Handlungsempfehlung zurückzuführen ist.

Dass dies bei der Nutzung von Gesundheits-Apps nicht immer der Fall ist, zeigen verschiedene Studien. So wurden Symptom-Checker-Apps im Ersteinschätzungsprozess von Krankheitssymptomen (Triage) kritisch bewertet [52]. Viele der untersuchten Apps zogen auch unwahrscheinliche Diagnosen mit in Betracht, was insbesondere ängstliche Patienten unnötig verunsichern kann. Auch Apps zur Einschätzung von Muttermalen führten zu vielen falschen Einschätzungen mit potenziell negativen Folgen für die Nutzer [53]. Das jüngste Beispiel einer beliebten, weitverbreiteten Blutdruck-App, die für ihre Nutzer ein erhebliches Sicherheitsrisiko darstellt, misst mit der Kamera des Smartphones den Blutdruck. Sie war bis zu ihrem Marktrückruf eine der 50 am meisten verkauften Gesundheits-Apps in Apples Store iTunes. Die ausgegebenen Messwerte waren zu niedrig, sodass Nutzer ihre Behandlungsbedürftigkeit nicht erkennen konnten [54]. Auch die immer beliebter werdenden Fitness-Tracker [3, 55], die z. B. Bewegungs- und Schlafverhalten aufzeichnen, zeigen bei Untersuchungen gravierende Sicherheitsmängel: Die erfassten Daten lassen sich vom Nutzer selbst oder von Dritten mit geringem Aufwand manipulieren [56]. Nur einer von 8 in einer Studie untersuchten Fitness-Tracker schützt den Nutzer vor der Aufzeichnung umfassender Bewegungsprofile [57].

Weder in Deutschland noch in den USA gibt es für Anbieter von Gesundheits-Apps Richtlinien, welche Informationen offenzulegen sind, damit Nutzer informiert entscheiden und sich vor Fehl- oder Falschinformation schützen können [16, 58, 59]. Erst wenige Fälle von irreführender Werbung hatten bisher rechtliche Konsequenzen: 2 Medizin-Apps zur Diagnose von gefährlichen Muttermalen und 2 Apps, die nach Angaben der Hersteller die Symptome von Akne verbessern sollten, sowie eine App, die vorgab die Sehkraft des Nutzers zu verbessern, wurden bisher in den USA wegen irreführender Werbung von der amerikanischen Bundesbehörde für Wettbewerb und Verbraucherschutz FTC (Federal Trade Commission) verklagt. Mit den Klagen waren hohe Geldstrafen verbunden, die Anbieter wurden zur Unterlassung der falschen Werbeaussagen verpflichtet [60].

\section{Regulierungsansatz in den USA:}

Auch nach den jüngsten Empfehlungen der amerikanischen Gesundheitsbehörde FDA bezüglich Wellnessprodukten [59] ist in 


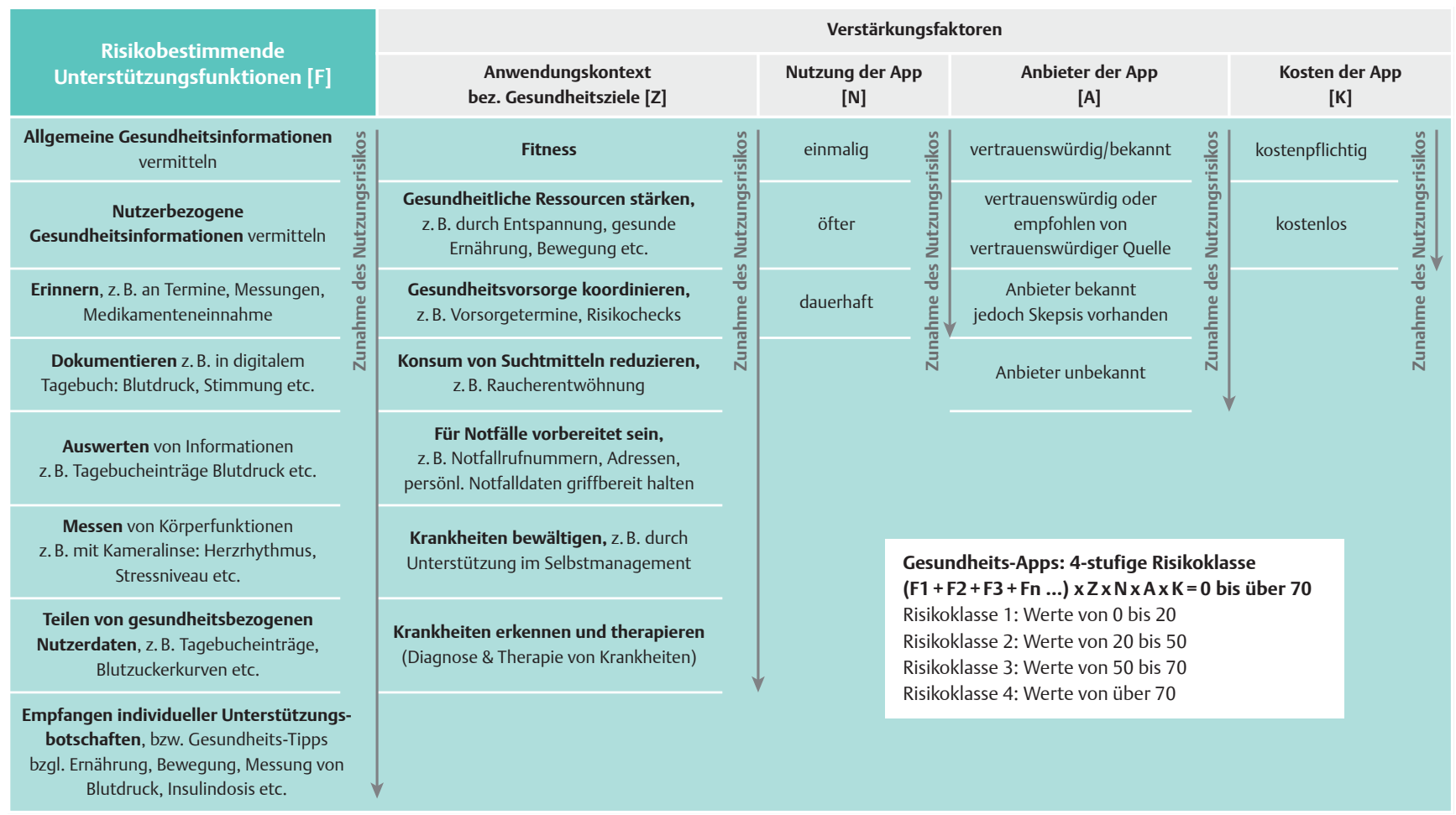

Abb. 3 Klassifizierung von Gesundheits-Apps in Risikoklassen: risikobestimmende Faktoren zur Berechnung der Risikoklasse [63].

den USA für Gesundheits-Apps weiterhin grundsätzlich keine Zulassung erforderlich. Die jüngste FDA-Empfehlung erklärt anhand vieler Beispiele, wann eine Zulassung benötigt wird und wann nicht. Entsprechende Empfehlungen für Deutschland bietet das Bundesinstitut für Arzneimittel und Medizinprodukte [16].

\section{Datensicherheit}

Sicherheit umfasst auch die Verhinderung von Schäden, die mit der Verletzung von Persönlichkeitsrechten des App-Nutzers einhergehen, wenn z. B. persönliche Gesundheitsdaten aus der App fahrlässig oder absichtlich in falsche Hände geraten und zum Nachteil des Anwenders von Dritten genutzt werden. Das Recht auf informationelle Selbstbestimmung, in Deutschland grundgesetzlich verbrieft (Grundgesetz Art. 2 Abs.1, Art. 1 Abs. 1), bildet die Basis für weitreichende Datenschutzrechte. Das Bundesdatenschutzgesetz (BDSG) definiert die geltenden Datenschutzgrundsätze wie Direkterhebung, Datensparsamkeit, Zweckbindung, anonyme und pseudonyme Nutzung und regelt damit den Schutz bzw. die Nutzungsvoraussetzungen für anonymisierte, pseudonymisierte und personenbezogene Gesundheitsdaten (BDSG §3, Abs. 1). Empfehlungen zur praktischen Umsetzung dieser Datenschutzrechte sollen App-Entwickler Orientierung geben [61].

Die Europäische Kommission entwickelt derzeit einen sog. Code of Conduct für Hersteller von Medizin-Apps, die gesundheitsbezogene Daten erfassen, verarbeiten bzw. verwalten [62]. Sie beschreibt, wie App-Entwickler die Regeln einhalten können, um den Schutz und die Sicherheit personenbezogener Daten auf Basis bestehender Datenschutzrechte zu gewähren.
Vertrauenswürdige Anbieter können sich freiwillig zur Einhaltung dieser Maßnahmen verpflichten. Sie sollen dann in einem Verzeichnis gelistet werden, das über die Website der Europäischen Kommission zugänglich gemacht werden soll.

\section{Risikoeinschätzung von Gesundheits- Apps}

Nutzen und Risiken von Gesundheits-Apps können sich sehr deutlich unterscheiden, abhängig davon, wofür und wie die App vom Anwender genutzt wird und von wem diese App zur Verfügung gestellt wird. Das empirisch entwickelte Konzept der Einteilung von Gesundheits-Apps in Risikoklassen [63] basiert auf der Analyse von über 6000 Gesundheits-Apps. Bei der Ermittlung des potenziellen Risikos, das für den Nutzer von der Anwendung einer Gesundheits-App ausgeht, werden folgende Faktoren beleuchtet:

- Art und Umfang der Unterstützungsfunktionen der App

- vorrangiges Gesundheitsziel, das mit der App verfolgt wird

- Häufigkeit der Nutzung

- Bekanntheit und Vertrauenswürdigkeit des Anbieters

- Finanzierungskonzept der App

\section{Unterstützungsfunktionen}

Abhängig von Art und Anzahl der Unterstützungsfunktionen und den Möglichkeiten, diese individualisiert nutzen zu können, erfassen, verwalten, analysieren oder teilen Apps gesundheitsbezogene Daten in mehr oder weniger großem Umfang: Je mehr Informationen die App aus der Lebenswelt des Nutzers er- 
fasst, umso eher ist sie in der Lage, individualisierte Handlungsempfehlungen und motivierende Botschaften zu liefern und damit in jeder Lebenssituation, rund um die Uhr, an 7 Tagen in der Woche Unterstützung zu bieten. Das erhöht den Nutzen der App für den Anwender. Andererseits steigt auch das Schadensmaß, wenn Daten z. B. unberechtigt genutzt oder Handlungsempfehlungen falsch ausgegeben werden. Insbesondere, wenn Daten über die App auch mit Dritten geteilt werden können, sind besondere Schutzmaßnahmen beim Versenden oder Speichern erforderlich, um unberechtigten Zugriff zu verhindern. Im Gegensatz dazu kann eine App, mit der sich lediglich Informationen abrufen lassen ohne jeden Bezug zu persönlichen Daten des Nutzers, wenig Schaden anrichten, das Risiko für den Anwender ist vergleichsweise gering.

\section{Gesundheitsziele}

Die gesundheitliche Zielsetzung, die mit einer App erreicht werden soll, fließt in die individuelle Risikobetrachtung ein, weil sie sich unmittelbar auf die Sensibilität der Daten auswirkt, die mit der App verwaltet oder ausgewertet werden. Sie beeinflusst auch den Stellenwert, den Auswertungen oder Empfehlungen der App für den Nutzer bekommen. Geht es z. B. um die Berechnung oder die Anzeige der Grenzwerte für Bluthochdruck, Blutzucker oder die Steuerung von Gerinnungsfaktoren durch Patienten, werden etwaige Fehler in den Berechnungen therapierelevant und können möglicherweise gravierende Folgen für die Gesundheit des Nutzers haben. Errechnet eine App im Gegensatz dazu auf Grundlage der gelaufenen Schritte die verbrannten Kalorien eines gesunden Anwenders falsch, ist die Gesundheitsgefährdung dieser Fehlfunktion vergleichsweise gering.

Auch die Bedeutung des Schutzes der von der App erfassten Daten verändert sich mit den Gesundheitszielen: Wird eine App z. B. als Stimmungstagebuch von einem Patienten mit Multipler Sklerose genutzt, verwaltet sie im Vergleich zu einer Vorsorgeoder Fitness-App viel sensiblere Daten. Was, wenn die Diagnose, die der Betroffene bisher geheim gehalten hat, in falsche Hände gerät und Nachteile drohen, z. B. durch den Arbeitgeber?

\section{Nutzungshäufigkeit}

Das Risiko, durch die Nutzung einer Gesundheits-App Schaden nehmen zu können, korreliert in der Regel auch damit, wie häufig die App genutzt wird. Referenz-Apps, mit denen Informationen von Zeit zu Zeit nachgeschlagen werden, z. B. ICD-10-Diagnosen oder medizinische Fachbegriffe werden weniger häufig genutzt, als z. B. ein Patiententagebuch, mit dem das verwaltete Datenvolumen mit jedem Tagebucheintrag wächst und damit auch das Risiko z. B. das dem Nutzer bei Datenverlust oder durch Fehlinformation droht. Im Einzelfall kann sich auch die einmalige Nutzung einer Fehl- oder Falschinformation fatal auf die Gesundheit oder Sicherheit des Nutzers auswirken.

\section{App-Anbieter}

Es fällt leichter, die Handlungsmotive eines Anbieters einzuschätzen, wenn der Nutzer diesen Anbieter kennt. Hat dieser einen Vertrauensbonus oder liegen bekannte Interessenkon- flikte vor, die zu einer gewissen Wachsamkeit aufrufen z. B. bei der Einordnung von Gesundheitsempfehlungen? Für die Mehrzahl der angebotenen Gesundheits-Apps ist der Anbieter unbekannt, nur wenige Angebote stammen von Krankenkassen, Arzneimittelherstellern oder Selbsthilfeorganisationen [64-66]. Bei unbekannten Herstellern ist grundsätzlich eine erhöhte Vorsicht ratsam, weil weder deren fachliche Expertise noch deren Handlungsmotive ohne Recherche eingeschätzt werden können.

\section{Kosten der App}

Die meisten Gesundheits-Apps werden kostenlos angeboten: 84,3 bzw. 89,6\% der deutschsprachigen Apps in Google Play in den Kategorien Medizin bzw. Gesundheit \& Fitness [45]. Nutzer sollten deshalb grundsätzlich damit rechnen, dass sie mit ihren Daten für die Nutzung der App bezahlen, weil Anbieter diese Daten unerlaubt nutzen und möglicherweise an Dritte verkaufen. Kostenlose Apps können sich auch durch den Abverkauf von Arznei- oder Nahrungsergänzungsmitteln refinanzieren, weshalb auch Vorsicht geboten ist, wenn Apps bestimmte Produkte empfehlen.

Fehlen Angaben, wie sich eine kostenlose App finanziert, muss der Nutzer mit Interessenkonflikten oder Verletzungen seiner Datenschutzrechte rechnen. Aus diesem Grund setzt sich der Nutzer immer einem erhöhten Risiko aus, wenn er sich für eine, in der Regel kostenlose App entscheidet.

\section{Ermittlung der Risikoklasse: Algorithmus}

Für die Ermittlung der Risikoklasse einer Gesundheits-App werden zunächst die risikorelevanten Unterstützungsfunktionen der App ermittelt, denen jeweils gemäß ihrem Gefährdungspotenzial ein Wert zugeordnet ist. Die Summe dieser Werte wird mit jeweils einem Faktor multipliziert, der das Risiko verstärken kann abhängig von der Art der Gesundheitsziele bzw. dem Anwendungskontext, der Vertrauenswürdigkeit oder Bekanntheit des App-Anbieters sowie dem Finanzierungskonzept der App. Nach diesem Algorithmus ergibt sich die Höhe der Risikoklasse auf einer 4-stufigen Skala von 0 bis über 70 (\Abb. 4).

Die folgenden beiden Beispiele zeigen, wie die Diabetes- und Entspannungs-Apps, die deutschsprachigen Verbrauchern derzeit in Google Play zur Verfügung stehen, auf die 4 Risikoklassen verteilen:

Diabetes-Apps: Zwei Drittel der untersuchten 51 kostenlosen, deutschsprachigen Diabetes-Apps gehören zu den beiden Risikoklassen 3 und 4, d.h. sie bieten dem Anwender weitreichende Unterstützung. Für den Nutzer ist die Anwendung dieser Apps daher auch mit erhöhten Risiken verbunden, weshalb eine Prüfung der Vertrauenswürdigkeit anhand der Herstellerangaben bei diesen Apps besonders ratsam ist [17].

Entspannungs-Apps: Ganz anders zeigt sich das Bild bei Apps, die Nutzer bei seelischer oder körperlicher Entspannung unterstützen wollen. Lediglich $10 \%$ der untersuchten Apps zählen demnach zu den Risikoklassen 3 und 4. Die meisten dieser Apps holen keine Daten von den Nutzern ein, sie fragen weder Gesundheitszustand noch Lebensgewohnheiten der Nutzer ab. Das Risiko, das mit der Nutzung dieser Apps verbunden ist, ist daher vergleichsweise gering ( $\triangleright$ Abb. 5 ). 


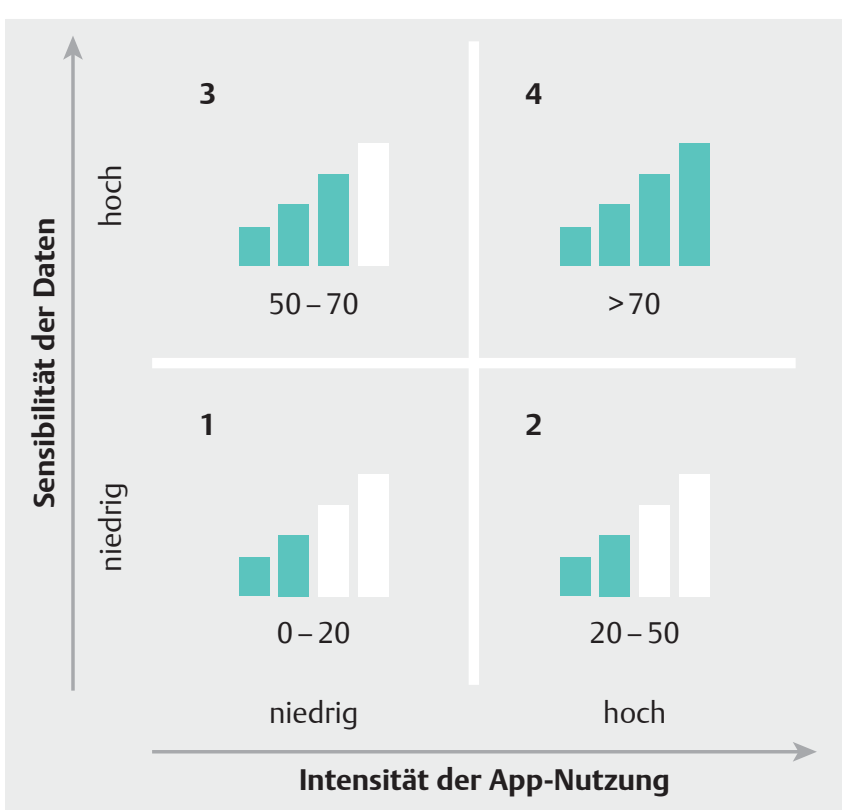

Abb. 4 Risikoklassifizierung von Gesundheits-Apps. Matrix zur Einordnung [63].

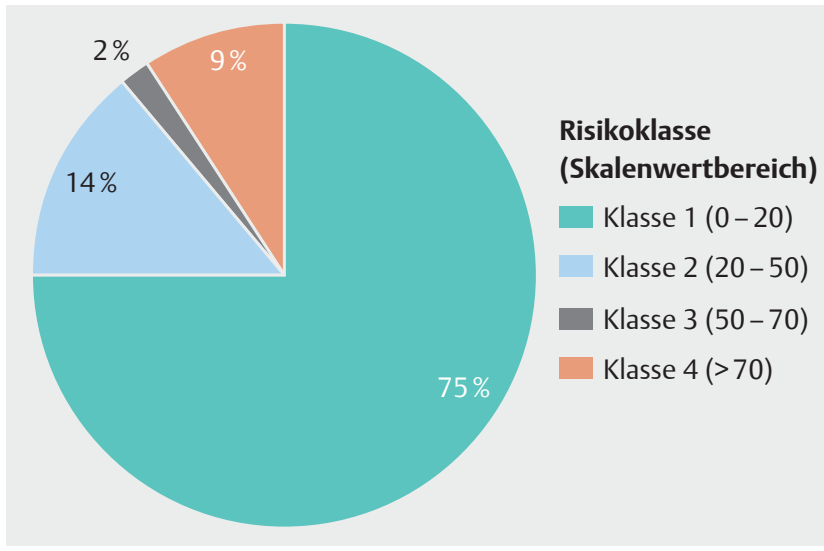

Abb. 5 Screening Entspannungs-Apps 06/2016, n=55. Prozentuale Verteilung in Risikoklassen 1 bis 4 [67].

Je höher die Risikoklasse einer Gesundheits-App, umso höher ist in der Regel auch der potenzielle Nutzen für den Anwender - vorausgesetzt die App arbeitet korrekt und schützt die Privatsphäre bzw. die personenbezogenen Daten des Nutzers. Nutzer sollten deshalb Apps mit hoher Risikoklasse besonders sorgfältig prüfen, damit sie ihr die persönlichen Gesundheitsdaten anvertrauen und sich auf die Berechnungen oder Empfehlungen der App verlassen können.

Das Konzept der Risikoklassen lässt sich auf jede Gesundheits-App anwenden. Die Kategorisierung von GesundheitsApps in Risikoklassen und die Möglichkeit für Verbraucher, diese selbst ermitteln zu können, stärkt die Souveränität bei der selbstbestimmten Auswahl und verbessert den Schutz von Verbrauchern [68]. Gerade Anbieter von Gesundheits-Apps mit hoher Risikoklasse sollten ihrer besonderen Verantwortung Rechnung tragen, in dem sie Nutzer möglichst umfassend, in allen 7 Kriterien gemäß HealthOn-Ehrenkodex informieren, damit eine Einschätzung der Vertrauenswürdigkeit ihrer Apps möglich ist. Das Beispiel der Diabetes-Apps zeigt, dass die Angaben der Anbieter sehr lückenhaft sind [17], obwohl zwei Drittel der Apps zur Risikoklasse 3 und 4 zählen.

\section{Ehrenkodex: Qualitäts- und Transparenzkriterien}

Die 7 Kriterien des HealthOn-Ehrenkodex orientieren sich am Konzept der Empfehlungen „Gute Praxis Gesundheitsinformationen“ [26] sowie an den Kriterien der Stiftung Health on the Net, die seit 20 Jahren Webseiten mit vertrauenswürdigen, gesundheitsbezogenen Inhalten mit dem HON-Prüfsiegel kenntlich macht [69]. Diese Kriterien wendet HealthOn bei der Analyse von Gesundheits-Apps seit 2011 an und verfolgt auf diese Weise die qualitative und quantitative Entwicklung der Gesundheits-Apps in den aus Public-Health-Perspektive relevanten Indikationen. Der Ehrenkodex versteht sich als Orientierungshilfe für Verbraucher und Patienten, die ohne fachlichen medizinischen oder gesundheitswissenschaftlichen Hintergrund anhand einfach überprüfbarer Kriterien eine informierte Entscheidung treffen können. Ziel ist es, das Risiko von Falsch- oder Fehlinformationen bzw. von Datenschutzverletzungen durch Überprüfung der Angaben des App-Anbieters einzuschätzen. Auch andere Ansätze zur Verbesserung der Qualität von Gesundheits-Apps und zur Förderung der Verbraucherorientierung, z.B. die App-Synopsis der medizinischen Hochschule Hannover [70] und das Factsheet Gesundheits-App des Aktionsforums Gesundheitsinformationssystem (afgis) e. V. [71], beziehen diese Kriterien ein ( $\triangleright$ Abb. $\mathbf{6}$ ).

Sind Angaben in Form einer Datenschutzerklärung vorhanden, die aufklärt wie Nutzer- bzw. Gesundheitsdaten geschützt werden? Gibt der Anbieter Aufschluss über die sog. Sachverständigkeit des Autors, der für die Korrektheit der gesundheitsbezogenen Aussagen in der App verantwortlich ist. Nennt er die Quellen, auf die sich z.B. Grenzwerte oder Empfehlungen beziehen oder Aussagen zur Wirksamkeit der App, z. B. wissenschaftliche Studien. Informiert die App darüber, wie sie sich finanziert bzw. warum die App dem Nutzer kostenlos zur Verfügung gestellt wird, um mögliche Interessenkonflikte erkennbar zu machen? Klärt die App auf über den Anbieter, nennt sie in einem Impressum Ansprechpartner, die z. B. auch im Fall von Haftungsansprüchen oder Datenschutzverletzungen adressiert werden können?

\section{Zusammenfassung und Ausblick}

Die aus Nutzersicht qualitätsbestimmenden Kriterien einer Gesundheits-App zu identifizieren [19, 20] und daraus Empfehlungen für die Entwicklung guter Gesundheits-Apps abzuleiten, ist sowohl für App-Entwickler, öffentliche Gesundheitsbehörden als auch für die Versorgungsforschung von großem Interesse, um Ressourcen zielgerichtet einsetzen zu können. Ebenso wichtig ist im weitgehend nicht regulierten, globalen Markt der Gesundheits-Apps die Sensibilisierung der Nutzer für Risiken, die mit der Nutzung von Gesundheits-Apps verbunden sind $[63,68]$. 


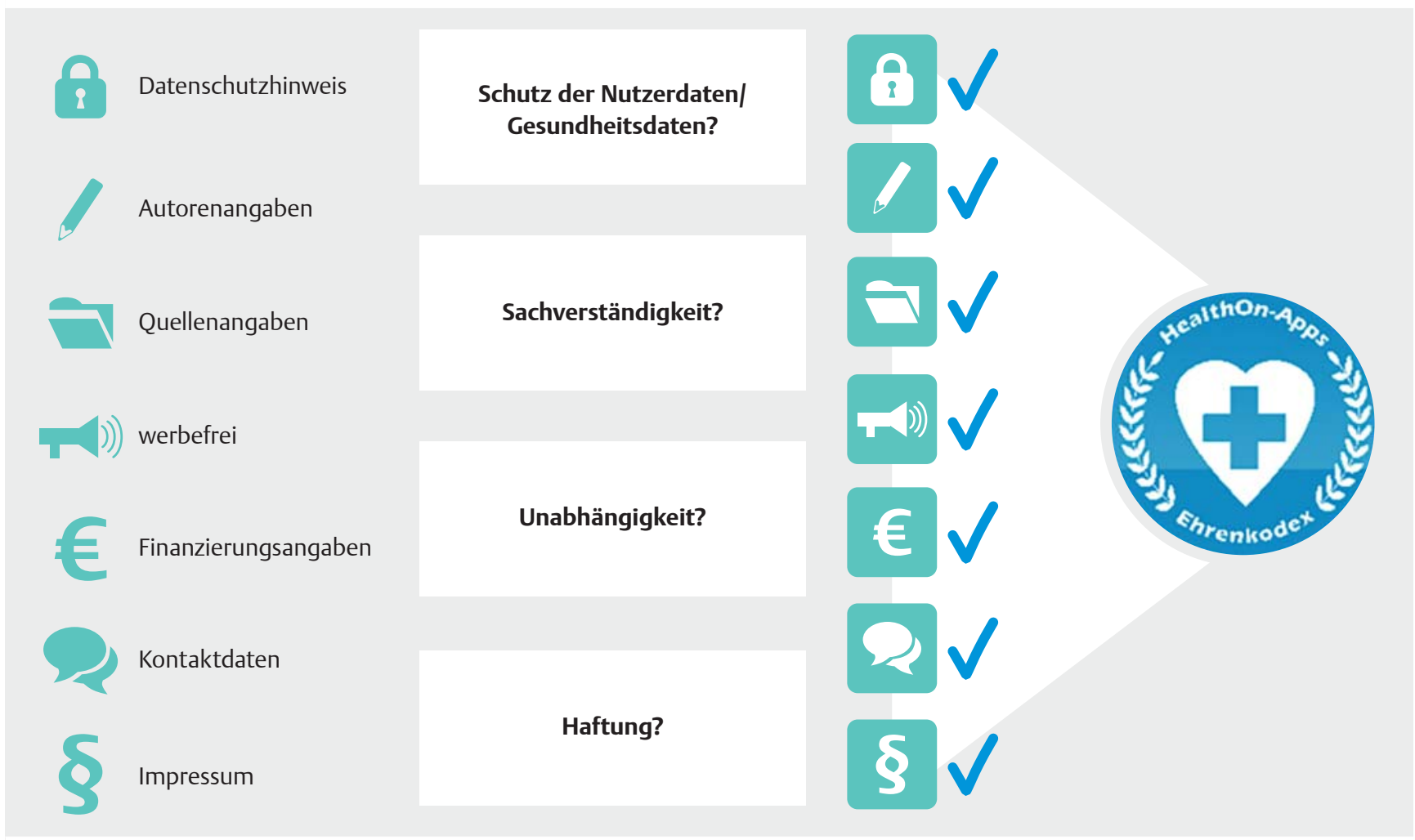

- Abb.6 Qualitäts- und Transparenzkriterien des HealthOn-App-Ehrenkodex für Gesundheits- und Medizin-Apps zur Orientierung für Verbraucher $[14,26,69]$.

Weil sich Risiken und Nutzen von Gesundheits- und MedizinApps abhängig von Art und Anzahl der Unterstützungsfunktionen und der Nutzung der App durch den Anwender deutlich unterscheiden [63], empfiehlt sich auch für Maßnahmen zum Schutz der Verbraucher und Patienten ein risikoadaptierter Ansatz. Werden Apps zur Therapie oder Diagnose von Krankheiten in Verkehr gebracht, ist bereits heute nach dem Medizinproduktegesetz eine CE-Kennzeichnung auf Basis eines EU-Konformitätsverfahrens erforderlich [16]. Ob und wenn ja für welche Gesundheits-Apps darüber hinaus zukünftig eine Regulierung angezeigt ist, wird unter Abwägung der Vor- und Nachteile für den Verbraucher sowie für den Marktstandort Deutschland zu entwickeln sein. Ein pragmatischer Schritt in Richtung Verbraucherschutz könnte die Pflicht zur Offenlegung aller Angaben sein, mit denen sich die Vertrauenswürdigkeit einer App für Verbraucher einschätzen lässt, z.B. gemäß Kriterien des HealthOn-Ehrenkodex. Ob sich dies auf Basis einer freiwilligen Selbstverpflichtung von App-Anbietern durchsetzen lässt, oder vom Gesetzgeber auf nationaler oder europäischer Ebene geregelt werden muss, wird die weitere Entwicklung zeigen.

Um digitale Gesundheitsanwendungen verantwortlich und selbstbestimmt nutzen zu können, wird die sog. Digital Literacy zu einer wichtigen Grundvoraussetzung sowohl für Verbraucher und Patienten als auch für Health Care Professionals [8]. Die Bereitstellung entsprechender Orientierungshilfen, mit denen sich zum einen die Risikoklassen ermitteln und zum anderen die Vertrauenswürdigkeit der Angebote einschätzen lassen [68], fördern die selbstbestimmte Nutzung von Gesundheits-
Apps durch Verbraucher und Patienten und helfen Therapeuten und Ernährungsberater, App-Empfehlungen abzusichern.

Gute digitale Angebote mit großem Nutzen für den Anwender benötigen ein hohes Maß an Zielgruppenorientierung und Anwenderfreundlichkeit (sog. Usability), denn nur dann werden sie genutzt und können gesundheitsförderlich wirken [41 46]. Die Entwicklung guter Apps erfordert deshalb die gemeinsame Anstrengung multidisziplinär zusammengesetzter Teams: Public Health Experten kennen den gesamtgesellschaftlichen Bedarf unter Berücksichtigung von Prävalenzdaten und Krankheitslast, Ärzte und Patientenvertreter können den individuellen Hilfebedarf und die Zugangsbarrieren von Nutzerzielgruppen verstehen, und Web-Designer und Usability-Experten sind in der Lage, die Expertise in einfach bedienbare, ansprechende Apps mit ganzheitlichem, wirksamem Unterstützungsansatz zu übersetzen. Die Versorgungsforschung ist aufgerufen, geeignete Methoden zu entwickeln, um den Nutzen von Apps als Bausteine innovativer, digitaler Versorgungsansätze zu evaluieren [72], und nachzuweisen, ob und in welchem Maße sich die mit der Digitalisierung der Gesundheit verknüpften Hoffnungen auf mehr Qualität, Effizienz und Patientenorientierung tatsächlich realisieren lassen.

Noch liegen wenig gesicherte Daten aus der Langzeitnutzung von Gesundheits-Apps vor, die deren langfristigen Nutzen wissenschaftlich belegen können [11-13]. Noch dominiert die Forderung nach besserem Verbraucher- und Patientenschutz, ohne konkrete Vorschläge zu benennen, wie das in einem dynamischen, globalen Markt realistisch umzusetzen ist, ohne das 
Innovationsklima zu trüben und damit nationale Märkte gegenüber USA und anderen außereuropäischen Märkten zu benachteiligen [12].

Damit die von Verbrauchern und Patienten über Apps und Wearables in Echtzeitdaten und rund um die Uhr aus ihrer Lebenswirklichkeit erfassten Daten zum Wohl des Einzelnen und darüber hinaus auch zur Verbesserung der gesundheitlichen Versorgung aller genutzt werden können, sind noch viele Fragen in einer gesamtgesellschaftlichen Diskussion zu klären: Wie sollen Patientendaten aus Apps und Wearables (z. B. Datenbrillen, Uhren, Fitness-Armbänder, intelligente Kleidung etc.) zukünftig genutzt werden? Welche Voraussetzungen sind an die Validität und den Austausch dieser Daten zu stellen, damit z.B. die Versorgungsforschung mithilfe dieser Daten bedeutungsvolle Zusammenhänge zwischen Verhalten, Umweltfaktoren und der Entwicklung von Zivilisationskrankheiten wie Diabetes oder Demenz erkennen kann? Welche Voraussetzungen müssen geschaffen werden, damit Gesundheits- und Medizin-Apps als sinnvolle Bausteine in die Regelversorgung integriert werden können? Für welche Patienten- und Verbraucherzielgruppen haben Apps einen besonders hohen Nutzen, wo ist der Bedarf besonders hoch? Mit welchen Instrumenten lassen sich Nutzen und Wirksamkeit dieser Anwendungen zukünftig evaluieren?

\section{Interessenkonflikt}

Dr. Kramer engagiert sich seit 2011 für den Aufbau der mittlerweile größten Bewertungsplattform für Gesundheits-Apps HealthOn in Deutschland und Europa. Die Plattform ist unabhängig und ohne Finanzierung durch öffentliche Fördergelder oder durch Sponsoren der Gesundheitswirtschaft entwickelt worden. Als Unternehmerin leitet Dr. Kramer sanawork, eine Agentur für Gesundheitskommunikation, und führt im Auftrag und in Kooperationen mit Universitäten und Krankenkassen Analysen sowie Marktforschungsstudien zu Gesundheits- und Medizin-Apps durch. Sie ist Präsidentin des Vereins HealthOn e. V., der die Qualität und Verbraucherorientierung von Gesundheits- und Medizin-Apps verbessern und den digitalen Wandel im Gesundheitswesen als gesamtgesellschaftlicher Aufgabe von hoher Bedeutung fördernd mitgestalten will.

\section{Literatur}

[1] Kramer U, Beyer S, Scherenberg V. GAPP Online-Befragung: Gesundheits-Apps zur Aufklärung, Prävention und Patientenführung. Derzeitige Nutzung, wahrgenommene Chancen, Risiken sowie Einschätzung von Maßnahmen zum Abbau potentieller Hürden. EU Consultation on Mobile Health; 2014: https://ec.europa.eu/digital-single-market/ en/news/summary-report-public-consultation-green-paper-mobilehealth (Zugriff am 20.10.2016)

[2] Kramer U, Zehner F. Diabetesmanagement mit Apps (DiMAPP). Chancen, Risiken, derzeitige \& zukünftige Nutzung, Einstellungen, Erfahrungen und Erwartungen von Betroffenen. Online-Befragung von Diabetikern. Diabetologie und Stoffwechsel 2016; 11: P118

[3] Bitkom und Bundesministerium der Justiz und für Verbraucherschutz. Fast ein Drittel nutzt Fitness-Tracker. 2016: https://www.bitkom.org/ Presse/Anhaenge-an-PIs/2016/Bitkom-Charts-PK-Safer-InternetDay-E-Tracker-und-Datenschutz-09-02-2016-final.pdf (Zugriff am 20.10.2016)
[4] Milani R et al. The role of Technology in Chronic Disease Care. Progress in Cardiovascular Diseases 2016: DOI: 10.1016/j.pcad.2016. 01.001

[5] Schroeder SA, Shattuck L. We can do better - improving health of the American people. The New England Journal of Medicine 2007; 357: $1221-1228$

[6] Greene J et al. When patient activation levels change, health outcomes and costs change, too. Health Affairs 2015; 34: 431-437

[7] Plass D et al. Trends in disease burden in Germany - results, implications and limitations of the Global Burden of Disease Study. Dtsch Arztebl Int 2014; 111: 629-638

[8] Europäische Kommission. Green Paper von Mobile Health (mHealth). https://ec.europa.eu/digital-single-market/en/news/green-papermobile-health-mhealth (Zugriff am 21.10.2016)

[9] Rutz M, Kühn D, Dierks ML. Kapitel 6. Gesundheits-Apps und Diagnostik \& Therapie. In: Albrecht U-V Hrsg. Chancen und Risiken von Gesundheits-Apps (CHARISMHA).Medizinische Hochschule Hannover; 2016: 136 -159. urn:nbn:de:gbv:084-16040811, 301; http:// www.digibib.tu-bs.de/?docid=60011

[10] Europäische Kommission. Summary Report on the Public Consultation on the Green Paper on Mobile Health. 2015: https://ec.europa. eu/digital-single-market/en/public-consultation-green-paper-mobile-health (Zugriff am 11.10.2016)

[11] Lucht M, Boeker M, Donath J, Güttler J, Leinfelder D, Kramer U. Gesundheits- und Versorgungs-Apps. Hintergründe zu deren Entwicklung und Einsatz. Freiburg: Universitätsklinikum Freiburg und sanawork Gesundheitskommunikation; https://www.tk.de/centaurus/ servlet/contentblob/724464/Datei/143238/Studie-Gesundheitsund-Versorgungs-Apps.pdf (Zugriff am 20.10.2016)

[12] Albrecht U-V. Kapitel Kurzfassung. In: Albrecht U-V, Hrsg. Chancen und Risiken von Gesundheits-Apps (CHARISMHA).Medizinische Hochschule Hannover; 2016: 14-47. http://www.digibib.tu-bs.de/ ?docid=60004 (Zugriff am 20.10.2016)

[13] Gigerenzer G, Schlegel-Matthies K, Wagner G. Digitale Welt und Gesundheit. eHealth und mHealth - Chancen und Risiken der Digitalisierung im Gesundheitsbereich. Veröffentlichung des Sachverständigenrats für Verbraucherfragen. http://www.bmjv.de/SharedDocs/ Artikel/DE/2016/01192016_Digitale_Welt.html (Zugriff am 11.10.2016)

[14] Scherenberg V, Kramer U. Schöne neue Welt: Gesünder mit HealthApps? Hintergründe, Handlungsbedarf und schlummernde Potenziale. In: Jahrbuch Healthcare Marketing. Hamburg: New Business Verlag; 2013: 115-119

[15] HealthOn. Gesundheits-, Medizin-Apps, Apps als Medizinprodukt? Definition \& Relevanz. https://www.healthon.de/de/2016/03/10/ gesundheits-medizin-apps-apps-als-medizinprodukt-definitionrelevanz (Zugriff am 21.10.2016)

[16] Bundesinstitut für Arzneimittel und Medizinprodukte (BfArm). Orientierungshilfe Medical Apps. 2015: http://www.bfarm.de/DE/Medi zinprodukte/Abgrenzung/medical_apps/_node.html (Zugriff am 20.10.2016)

[17] HealthOn. Diabetes-Apps: Angebot und Nachfrage wachsen, Qualität steigt. 08/2016. https://www.healthon.de/de/2016/08/29/diabetesapps-angebot-und-nachfrage-wachsen-qualit\%C3\%A4t-steigt (Zugriff am 20.10.2016)

[18] YouGov Studie. Quantified Health 2015. https://yougov.de/loesun gen/ueber-yougov/presse/presse-2015/pressemitteilung-self-tra cking-rund-jeder-dritte-wurde-gesundheitsbezogene-daten-ankrankenversicherer-weitergeben/ (Zugriff am 20.10.2016)

[19] Stoyanov SR, Hides L, Kavanagh DJ et al. Development and Validation of the User Version of the Mobile Application Rating Scale (uMARS). JMIR Mhealth Uhealth 2016; 4: e72 
[20] Stoyanov SR, Hides L, Kavanagh D] et al. Mobile App Rating Scale: A New Tool for Assessing the Quality of Health Mobile Apps. JMIR Mhealth Uhealth 2015; 3: e27

[21] GKV-Spitzenverband, Hrsg. Leitfaden Prävention. Handlungsfelder und Kriterien des GKV-Spitzenverbandes zur Umsetzung von $\S \S 20$ und 20 a SGB V vom 21. Juni 2010. Basel/Berlin: Prognos AG

[22] GKV-Spitzenverband, Hrsg. Gemeinsame Empfehlung zur Förderung und Durchführung von Patientenschulungen auf der Grundlage von $\S 43$ Abs. 1 Nr. 2 SGB V.https://www.gkv-spitzenverband.de/media/ dokumente/krankenversicherung_1/rehabilitation/patientenschu lung/2015_05_07_GEP_allgemeiner_Teil_4_2015.pdf

[23] Bundeszentrale für gesundheitliche Aufklärung. Gesundheitsförderung konkret. Band 13: Qualitätskriterien für Maßnahmen der Gesundheitsförderung und Primärprävention von Übergewicht bei Kindern und Jugendlichen 2010: http://www.bzga.de/botmed_ 60649130.html (Zugriff am 20.10.2016)

[24] Bundeszentrale für gesundheitliche Aufklärung. Gesundheitsförderung konkret. Band 5: Kriterien guter Praxis in der Gesundheitsförderung bei sozial Benachteiligten. 5. Auflg. 2011: http://www.bzga.de/ botmed_60645000.html

[25] Bundeszentrale für gesundheitliche Aufklärung. Leitfaden Qualitätskriterien für Planung, Umsetzung und Bewertung von gesundheitsfördernden Maßnahmen mit dem Fokus auf Bewegung, Ernährung und Umgang mit Stress. 2012: http://www.bzga.de/infomaterialien/ ernaehrung-bewegung-stressbewaeltigung/leitfaden-qualitaetskri terien/ (Zugriff am 20.10.2016)

[26] Deutsches Netzwerk Evidenzbasierter Medizin e. V. Gute Praxis Gesundheitsinformation. Zeitschrift für Evidenz, Fortbildung, Qualität im Gesundheitswesen 2009; 104: 66-68

[27] Scherenberg V, Kramer U. Krankenkassen-Apps: Hintergründe, Status quo und Herausforderungen. Monitor Versorgungsforschung 2014; 5: $51-53$

[28] Green LW, Kreuter MW. Health promotion planning: An educational and environmental approach. Mountain View, CA: Mayfield Pub. Co; 1991

[29] Mevvy, IMS Health, AppScript in: Patient adoption of mHealth 06/ 2016

[30] Deutsches Netzwerk Versorgungsforschung e. V. AG Digital Health. Ziele und Aufgaben. http://www.netzwerk-versorgungsforschung. de/index.php?page=ag-digital-health (Zugriff am 20.10.2016)

[31] Albrecht U-V, von Jan U, Pramann O, Fangerau H. Kapitel 7. Gesundheits-Apps im Forschungskontext. In: Albrecht U-V, Hrsg. Chancen und Risiken von Gesundheits-Apps (CHARISMHA). Medizinische Hochschule Hannover; 2016: 160 - 175 urn:nbn:de: gbv:08416040811320. http://www.digibib.tu-bs.de/?docid=60013 (Zugriff am 20.10.2016)

[32] Institut für Qualität und Wirtschaftlichkeit im Gesundheitswesen. Allgemeine Methoden: Version 4.2. https://www.iqwig.de/download/ IQWiG_Methoden_Version_4-2.pdf (Zugriff am 20.10.2016)

[33] Hüsing A. 5 Apps, für die Krankenkassen ein Rezept ausstellen. https://www.deutsche-startups.de/2016/06/22/5-apps-fuer-diekrankenkassen-ein-rezept-ausstellen/ (Zugriff am 20.10.2016)

[34] Stein A et al. Clinical trial on tonal tinnitus with tailor-made notched music training. BMC Neurology 2016; 16: 38

[35] GfK Umfrage. Jeder vierte deutsche Internetnutzer setzt auf Gesundheits-Apps oder Fitness-Tracker. 29.09.2016. http://www.gfk.com/ de/insights/press-release/jeder-vierte-deutsche-internetnutzersetzt-auf-gesundheits-apps-oder-fitness-tracker/ (Zugriff am 20.10.2016)

[36] Zickuhr K. Pew Research Internet Project: tablet ownership 2013. http://www.pewinternet.org/2013/06/10/tablet-ownership-2013/ (Zugriff am 20.10.2016)
[37] Smith A. Pew Research Internet Project: smartphone ownership. 2013: http://www.pewinternet.org/2013/06/05/smartphone-ownership-2013/ (Zugriff am 20.10.2016)

[38] Institute of Medicine. Health literacy: a prescription to end confusion. Washington (DC): Institute of Medicine; 2004: https://www.nap.edu/ catalog/10883/health-literacy-a-prescription-to-end-confusion (Zugriff am 20.10.2016)

[39] Schempp N, Jung C, Seidel J, Strippel H. Präventionsbericht 2012. Leistungen der gesetzlichen Krankenversicherung: Primärprävention und Betriebliche Gesundheitsförderung. Berichtsjahr 2011. Essen, Berlin: MDS - Medizinischer Dienst des Spitzenverbandes Bunde der Krankenkassen e. V.; GKV Spitzenverband;

[40] Cho J, Park D, Lee HE. Cognitive factors of using health apps: systematic analysis of relationships among health consciousness, health information orientation, eHealth literacy, and health app use efficacy. J Med Internet Res 2014; 16: e125 CrossRef PubMed

[41] Schillinger D, Grumbach K, Piette J et al. Association of health literacy with diabetes outcomes. JAMA 2002; 288: 475-482 CrossRef PubMed

[42] Broderick J, Devine T, Langhans E, Lemerise A, Lier S, Harris L. Designing health literate mobile apps: discussion paper. Washington (DC): Institute of Medicine; 2014: http://www.iom.edu/Global/Per spectives/2014/HealthLiterateApps.aspx (Zugriff am 20.10.2016)

[43] Mackert M, Mabry-Flynn A, Champlin S et al. Health Literacy and Health Information Technology Adoption: The Potential for a New Digital Divide. J Med Internet Res 2016; 18: e264

[44] Caburnay CA, Graff K, Harris JK et al. Evaluating Diabetes Mobile Applications for Health Literate Designs and Functionality, 2014. Prev Chronic Dis 2015; 12: 140433

[45] Sarkar U, Gourley G, Lyles C et al. Usability of Commercially Available Mobile Applications for Diverse Patients. Journal of General Internal Medicine; published online July 14, 2016

[46] Arnhold M, Quade M, Kirch W. Mobile applications for diabetics: a systematic review and expert-based usability evaluation considering the special requirements of diabetes patients age 50 years or older. J Med Internet Res 2014; 16: e104 CrossRef PubMed

[47] Health-App Dashboard. Anzahl der Apps in den Kategorien Gesundheit und Fitness sowie Medizin, weltweit in Google Play und Apple iTunes. https://www.healthon.de/health-app_dashboard (Zugriff am 20.10.2016)

[48] HealthOn. Die 100 Top Gesundheits-Apps: Einsatzgebiete, Unterstützungsfunktionen \& Qualität. Auf https://www.healthon.de/de/ 2015/07/16/die-100-top-gesundheits-apps-einsatzgebiete-unterst\% C3\%BCtzungsfunktionen-qualit\%C3\%A4t (Zugriff am 28.09.2016)

[49] Altgeld Th, Kolip P. Konzepte und Strategien der Gesundheitsförderung. In: Hurrelmann K, Koltz T, Haisch J, Hrsg. Lehrbuch der Prävention und Gesundheitsförderung. Bern: Hans Huber; 2004: 41 - 51

[50] Farago P. Flurry Analytics 2012. App Engagement: The Matrix Reloaded. 2012: Oct 22. Available from: http://flurrymobile.tumblr.com/ post/113379517625/app-engagement-the-matrix-reloaded (Zugriff am 20.10.2016)

[51] Flurry Analytics 2016. Enter the Matrix: App Retention and Engagement. May 2016: http://flurrymobile.tumblr.com/post/144245637 325/appmatrix (Zugriff am 20.10.2016)

[52] Semigran HL, Linder JA, Gidengil C et al. Evaluation of symptom checkers for self diagnosis and triage: audit study. BMJ 2015; 351: h3480

[53] Wolf J, Moreau J, Akilov O et al. Diagnostic Inaccuracy of Smarthphone Applications for Melanoma Detection. JAMA Dermatol 2013; 149: $422-426$

[54] Plante TB, Urrea B, MacFarlane ZT et al. Validation of the Instant Blood Pressure Smartphone App. JAMA Intern Med 2016; 176: 700 - 702 
[55] ICD Research 2016. Worldwide Wearables Market Increases 67.2\% Amid Seasonal Retrenchment, According to IDC.http://www.idc.com/ getdoc.jsp?containerld=prUS41284516 (Zugriff am 20.10.2016)

[56] Sadeghi A-R. Fitness-Tracker schwächeln: Informatiker der TU Darmstadt decken schwere Sicherheitsmängel auf. 2016: https://www.tudarmstadt.de/vorbeischauen/aktuell/einzelansicht_157888.de.jsp (Zugriff am 20.10.2016)

[57] Open Effect \& Citizen Lab University Toronto. Every Step you Fake. A Comparative Analysis of Fitness Tracker Privacy and Security 2016. https://openeffect.ca/reports/Every_Step_You_Fake.pdf (Zugriff 20.10.2016)

[58] Food and Drug Administration (FDA). Mobile Medical Applications. Guidance for Industry and Food and Drug Administration Staff. February 9, 2015: http://www.fda.gov/downloads/MedicalDevices/ .../UCM263366.pdf (Zugriff am 20.10.2016)

[59] Food and Drug Administration (FDA). General Wellness: Policy for Low Risk Devices. Guidance for Industry and Food and Drug Administration Staff.. July 29 2016: http://www.fda.gov/downloads/medicalde vices/deviceregulationandguidance/guidancedocuments/ ucm429674.pdf (Zugriff am 20.10.2016)

[60] HealthOn. Klage gegen Medizin-App, die Sehkraft verbessern soll. 29.09.2015: https://www.healthon.de/de/2015/09/29/klage-gegenmedizin-app-die-sehkraft-verbessern-soll

[61] Düsseldorfer Kreis. Orientierungshilfe Datenschutzanforderungen an App-Entwickler und App-Anbieter. Ansbach: Bayerisches Landesamt für Datenschutzaufsicht; 2014: https://www.Ida.bayern.de/media/ oh_apps.pdf (Zugriff am 20.10.2016)

[62] European Commission. Code of Conduct on privacy for mHealth. apps has been finalised 07.06.2016: https://ec.europa.eu/digital-singlemarket/en/news/code-conduct-privacy-mhealth-apps-has-beenfinalised

[63] HealthOn. Risikoskala für Gesundheits-Apps.https://www.healthon. de/de/risikoklasse-fuer-gesundheits-apps (Zugriff am 20.10.2016)
[64] HealthOn. Gesundheits-Apps gesetzlicher Krankenkassen: Wie beliebt sind sie? 17.02.2016: https://www.healthon.de/de/2016/02/17/ gesundheits-apps-gesetzlicher-kassen-wie-beliebt-sind-sie (Zugriff am 20.10.2016)

[65] HealthOn Testdatenbank. Stand. Oktober 2016. Anbietergruppe: Selbsthilfe.https://www.healthon.de/de/testberichte?f[0]=field_product\%253Afield_anbietergruppe\%253Aname\%3ASelbsthilfe (Zugriff am 20.10.2016)

[66] HealthOn. Fast 30 Prozent mehr Krankenkassen- und Pharma-Apps. 14.04.2014: https://www.healthon.de/de/2014/04/14/fast-30-prozent-mehr-krankenkassen-pharma-appsPharma-Apps (Zugriff am 20.10.2016)

[67] Healthon. Entspannungs-Apps vs Diabtes-Apps: Risiko variiert erheblich. https://www.healthon.de/de/2016/10/24/entspannungs-appsvs-diabetes-apps-risiko-variiert-erheblich (Zugriff am 24.10.2016)

[68] HealthOn. Online Checkliste Gesundheits-Apps zur Ermittlung der Risikoklasse und Überprüfung der Herstellerangaben gemäß Healthon-Ehrenkodex. https://www.healthon.de/de/checkliste (Zugriff am 20.10.2016)

[69] Health on the Net Foundation. The HON Code of Conduct for medica and health Webs sites. http://www.hon.ch/HONcode/Patients/Con duct.html (Zugriff am 27.10.2016)

[70] Albrecht U-V. Transparency of Health-Apps for Trust and Decision Making. Eysenbach G, ed. Journal of Medical Internet Research 2013; 15: e277

[71] Aktionsforum Gesundheitsinformationssystem (afgis) e. V. Was muss man über Gesundheits-Apps wissen. 2012: https://www.afgis.de/ standards/gesundheits-app-fact-sheet/files/afgis_apps_web.pdf (Zugriff am 21.02.2017)

[72] Schrappe M. Versorgungsforschung braucht eine digitale Agenda. Monitor Versorgungsforschung 2016; 2: 52- 57 